\title{
Miniature Direct Electron Transfer Based Enzymatic Fuel Cell Operating in Human Sweat and Saliva
}

\author{
M. Falk ${ }^{1 *}$, D. Pankratov ${ }^{1}, 2$, L. Lindh ${ }^{3}$, T. Arnebrant ${ }^{1}$, S. Shleev ${ }^{1,4}$ \\ 1 Biomedical Science, Health and Society, Malmö University, 20506 Malmö, Sweden \\ 2 Bach Institute of Biochemistry, 119 071, Moscow, Russia \\ 3 Prosthetic Dentistry, Faculty of Odontology, Malmö University, 20506 Malmö, Sweden \\ ${ }^{4}$ Kurchatov NBIC Centre, National Research Centre "Kurchatov Institute", 123182 Moscow, Russia
}

Received March 10, 2014; accepted April 22, 2014; published online October 22, 2014

\begin{abstract}
We present data on operation of a miniature membrane-less, direct electron transfer based enzymatic fuel cell in human sweat and saliva. The enzymatic fuel cell was fabricated following our previous reports on miniature biofuel cells, utilizing gold nanoparticle modified gold microwires with immobilized cellobiose dehydrogenase and bilirubin oxidase. The following average characteristics of miniature glucose/oxygen biodevices operating in human sweat and saliva, respectively, were registered: 580 and $560 \mathrm{mV}$ opencircuit voltage, 0.26 and $0.1 \mu \mathrm{W} \mathrm{cm}$ power density at a cell voltage of $0.5 \mathrm{~V}$, with up to ten times higher power output at $0.2 \mathrm{~V}$. When saliva collected after meal ingestion was
\end{abstract}

used, roughly a two-fold increase in power output was obtained, with a further two-fold increase by addition of $500 \mu \mathrm{M}$ glucose. Likewise, the power generated in sweat at $0.5 \mathrm{~V}$ increased two-fold by addition of $500 \mu \mathrm{M}$ glucose.

Keywords: Enzymatic Fuel Cell, Microscale, Non-Invasive, Saliva, Sweat

\section{Supplementary Material}

Supporting Information is available from the Wiley Online Library or from the author.

\section{Introduction}

Biological fuel cells utilizing enzyme catalysts (enzymatic fuel cells, EFCs) have been envisioned as electrical power sources for self-contained implantable electronic devices [13]. EFCs have some great advantages due to the high specificity and activity of enzymes, and utilizing a direct electron transfer (DET) approach, can allow great simplification in the designed biodevice excluding the need of membranes and toxic mediators. Such EFCs can potentially be produced at low cost and have great possibilities for miniaturization. However, in vivo operation possesses some significant hurdles to overcome, since an implanted biodevices would require a long operational lifetime with many applications requiring surgery. Due to biocompatibility issues, with the implanted biodevice being affected by the hosts immune system [4] and the inherent short-term stability of enzymes, this can prove quite difficult. The actual in vivo performance of EFCs already tested (e.g. in Refs. [5-8]), which indeed only have been reported in a few cases, illustrate the difficulty of operation inside a living vertebrate, where the efficiency of the biodevices is limited by incomplete fuel oxidation and short lifetime because of quite limited enzyme stability or hampered by rather bulky design.

By avoiding actual implantation of the EFC, many of the issues concerning biocompatibility and stability could be avoided. This would entail biodevices operating in non-invasive contact situations, such as attachable, adhesive and/or floating biodevices operating in e.g. saliva, tears, and sweat, which lie in between in vivo and in vitro situations. Such wearable or attachable biodevices could largely be unexposed to the immune system, and by utilizing a cheap and flexible design the short stability would not be an issue, considering that the devices could easily be exchanged. By for example utilizing an EFC in sweat, such a biodevice could be used to power electronic skin patches [9]. Indeed, a recent study by Wang and co-workers [10] reported on a noninvasive epidermal EFC able to convert lactate in sweat into electrical energy,

["] Corresponding author, magnus.falk@mah.se 
a device which was envisioned as a potential power source for skin-worn electronic devices. The EFC was based on temporary transfer tattoos employing a mediator-based lactate oxidase bioanode combined with a platinum black based cathode. Interest has also been shown in utilizing sweat for non-invasive glucose sensing, where a correlation between sweat glucose and blood glucose have been shown in patients with diabetes [11].

Apart from sweat, saliva, and tears also offer an opportunity for non-invasive biomedical devices or other types of sensors [12], where an EFC potentially could be employed to generate power for a self-powered biodevice. We recently reported on a microscale EFC operating in human tears, where we suggested its use as a power source for bioelectronic contact lenses [13]. The EFC was fabricated utilizing gold nanoparticle (AuNP) modified microwires, employing a cellobiose dehydrogenase $(\mathrm{CDH})$ modified anode for glucose oxidation and a bilirubin oxidase (BOx) modified cathode for oxygen reduction. In this paper we exploit the same EFC design to investigate the performance in human sweat and saliva, and to compare the performance with samples collected at different times and with different glucose concentrations.

\section{Materials and Methods}

All chemicals were of analytical grade and used without further purification. Purified $\mathrm{CtCDH}$ and $\mathrm{MvBOx}$ were received as kind gifts from BOKU-University (Austria) and Amano Enzyme Inc. (Japan), respectively. Human physiological fluids were collected from healthy volunteers aging between 28 and 30 years old. Heat induced sweat was collected by scrapings from the back and arms at one occasion from a female donor. Unstimulated saliva was collected from a male donor over five different days, each day collecting both directly before lunch and roughly $15 \mathrm{~min}$ following the meal for a duration of roughly 10 min each time, as known in the arts [14]. Prior to measurements, the saliva samples collected during different days were pooled together, separating samples into collected before and after lunch. Additionally, $500 \mu \mathrm{M}$ glucose was added to some of the sweat and saliva samples collected after lunch. The ethical approval for the experiments was obtained in advance from the Lund ethical committee (LU 2010/649) and the Lund/Malmö ethical committee in the frame of EU Marie Curie project "Bioenergy" (PEOPLE-2013-ITN-607793).

A similar approach as reported previously was used to design the bioelectrodes [13], employing AuNP modified gold microwires with Myrothecium verrucaria (Mv)BOx and Corynascus thermophilus ( $\mathrm{Ct}) \mathrm{CDH}$ to create the biocathode and bioanode, respectively. Briefly, Au microwires with a diameter of $100 \mu \mathrm{m}$ were insulated using cellulose acetate (10 $\mathrm{mg} \mathrm{mL}^{-1}$ ) dissolved in pure acetone, leaving a length of roughly $3 \mathrm{~mm}$ un-insulated. Electrodes were thereafter polished with $0.05 \mu \mathrm{m}$ aluminum oxide powder and cleaned electrochemically by potential cycling in $0.5 \mathrm{M} \mathrm{H}_{2} \mathrm{SO}_{4}$. Concentrated AuNPs were subsequently applied to the surface of the cleaned electrodes $(\sim 17 \mathrm{~nm}$ in size, $c a .3 \times 1 \mu \mathrm{L})$, modifying roughly $1.5 \mathrm{~mm}$ of the working surface (projected [geometric] area of about $0.5 \mathrm{~mm}^{2}$ ) and electrochemically cleaned again. AuNPs modification increased the electrochemically active surface area around 100 times. The cathodes were prepared by incubation in a $2 \mathrm{mg} \mathrm{mL}^{-1} \mathrm{MvBOx}$ solution for $2 \mathrm{~h}$, thereafter washed with phosphate buffer. The anodes were incubated for $1 \mathrm{~h}$ in a mixed thiol solution (1:1 mixture of $1 \mathrm{mM}$ 4-aminothiophenol and $1 \mathrm{mM}$ 4-mercaptobenzoic acid), washed with ethanol, and incubated in $\mathrm{CtCDH}$ with gludaraldehyde crosslinker for $2 \mathrm{~h}\left(6 \mu \mathrm{L} 23 \mathrm{mg} \mathrm{mL}^{-1} \mathrm{CtCDH}+3 \mu \mathrm{L}\right.$ $2 \%$ glutaraldehyde). Finally, the anodes were carefully washed with phosphate buffer.

The $\mathrm{CtCDH}$ concentration on the electrode surface was

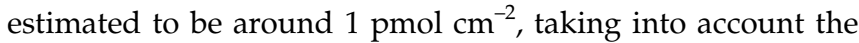
real surface area, corresponding to sub-monolayer coverage. The estimation was done following our previous report [15]. The MvBOx surface coverage could not be determined, as non-turnover signals from the enzyme were not observed.

Electrochemical characterization of the EFC was performed in a microcell constructed from a glass capillary with a volume of roughly $20 \mu \mathrm{L}$ and an inside diameter of $1.5 \mathrm{~mm}$, carried out using a $\mu$ Autolab Type III/FRA2 potentiostat/ galvanostat from Metrohm Autolab B.V. (The Netherlands). During all measurements the humidity was kept around $100 \%$ by placing the cell in a box to prevent evaporation of the electrolyte solutions. Basic EFC parameters were obtained using the bioanode as working electrode and the biocathode as a combined counter/reference electrode. The voltage was changed from open circuit voltage $(\mathrm{OCV})$ to lower voltage regions with a scan rate of $0.1 \mathrm{mV} \mathrm{s}^{-1}$, while recording the current in the circuit. The low scan rate eliminated charging/ discharging currents of the double layer in the obtained parameters. Stability tests were performed by chronoamperometry using an applied potential of $0.2 \mathrm{~V}$, where the EFCs produced the maximum power. Results averaged over 3-5 different EFCs for each measurement were used to estimate statistical parameters.

\section{Results and Discussion}

An overall scheme of the study is shown in Figure 1. A nanostructured miniature glucose/oxygen EFC was used to allow investigations in human sweat and saliva, only needing a very small sample volume $(-20 \mu \mathrm{L})$ to perform the measurements. Physiological fluids were collected as known in the arts, and by employing $\mathrm{CtCDH}$ to design the bioanode the glucose present in the sample could be utilized as fuel. Vice versa, by immobilizing $M v \mathrm{BO}$ at the biocathode oxygen was used as oxidant. Characterization of the individual bioelectrodes, as well as the complete EFC in simple buffers, are presented in the Supplementary Information (SI, Figures S1 and S2), and is also discussed in detail in our previous work [13]. 


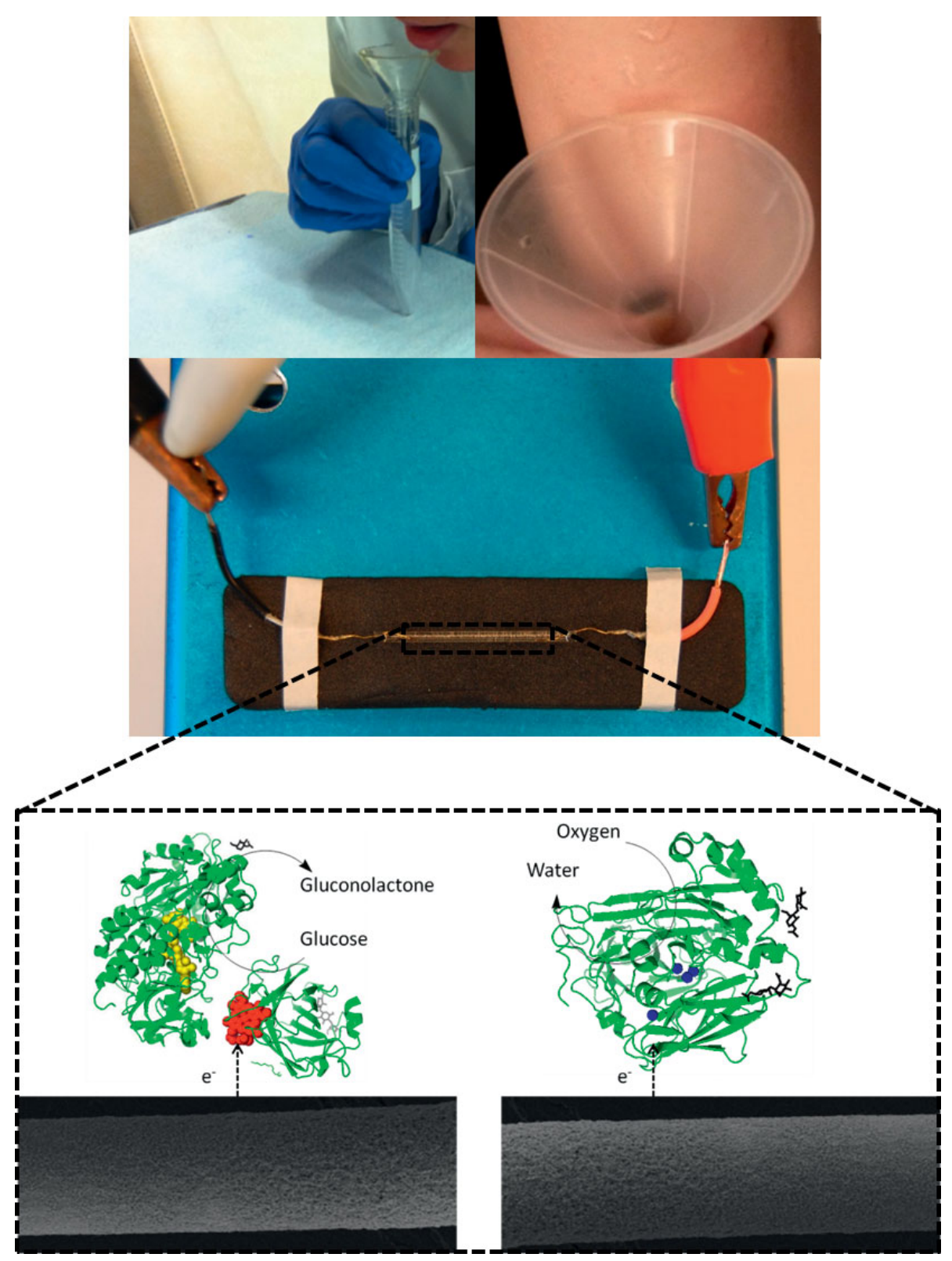

Fig. 1 Scheme over the design of the study. Note: the structure of $B O x$ was taken from the known crystal structure (PDB 2XLL) and CDH was designed using the cytochrome and the FAD domains of Phanerochaete chrysosporium $\mathrm{CDH}$, since the complete structure of the enzyme is still unavailable (PDB ID7D and INAA, respectively).

\subsection{Miniature EFC Operating in Human Sweat}

A typical response for the miniature glucose/oxygen EFC, when operating in human sweat, is shown Figure 2a, also displayed as power $v s$. current curve in SI, Figure S3A, and corresponding characteristics are summarized in Table 1 . In pure sweat (solid curves in Figure 2a), an average OCV of $580( \pm 5) \mathrm{mV}$ was recorded. The current output is fairly low and constant from the OCV down to around $0.3 \mathrm{~V}$, where a dramatic increase in the current is observed. The EFC recorded an average current output of $0.53( \pm 0.05) \mu \mathrm{A} \mathrm{cm}{ }^{-2}$ at $0.5 \mathrm{~V}$ and $6.0 \mu \mathrm{A}( \pm 5.1) \mathrm{cm}^{-2}$ at $0.2 \mathrm{~V}$. The low current output at high voltage, due to bioelectrocatalytic oxidation of glucose, can be explained by the very low concentration of glu- cose available in sweat, with large variation in the actual composition of the fluid. Sweat is known to be composed of highly variable amounts of electrolytes, carbohydrates, amino acids, organic acids, nitrogenous substances, and miscellaneous constituents, depending on, e.g. body region, age, diet, activity level, sampling technique, with a widely variable $\mathrm{pH}$ (2.1-8.2, median 5.3) and amount of glucose $(5.6-2,200 \mu \mathrm{M}$, median $170 \mu \mathrm{M})[16,17]$. At lower voltage (i.e. a high overpotential for the bioanode), the large current observed is originating from electrocatalytic oxidation of other compounds than glucose in the sweat due to the supporting layer of AuNPs [18]. This is the cause of the peculiar shape of the power curve, where two peaks can be observed due to the 

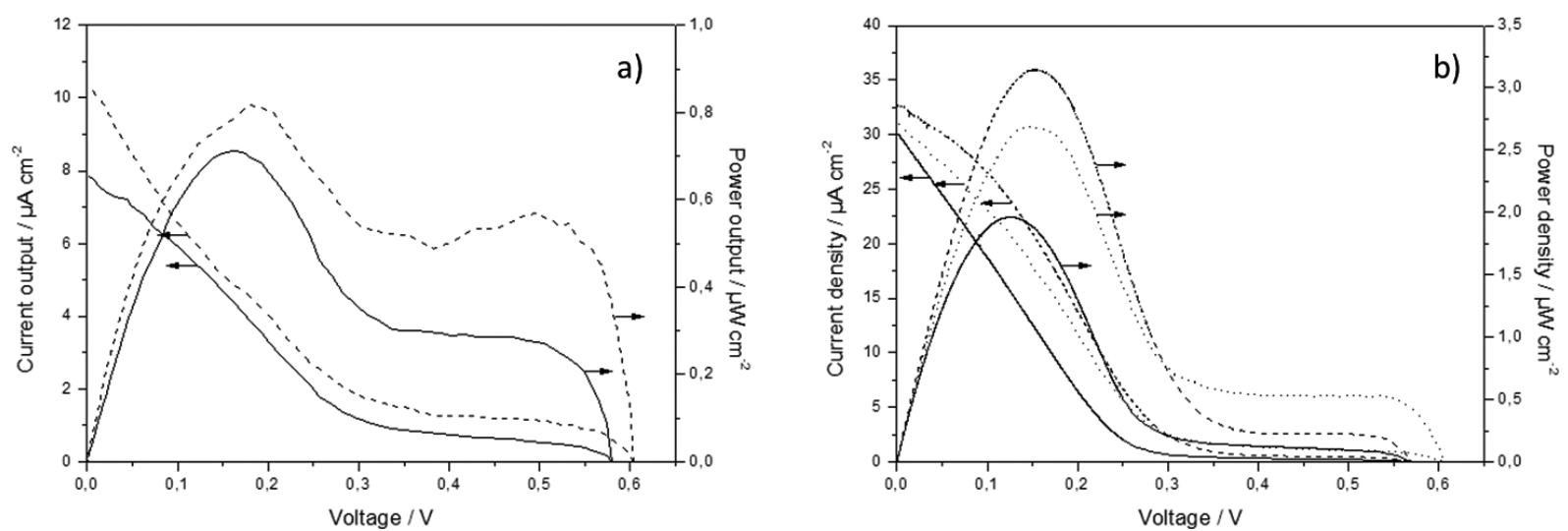

Fig. 2 Typical response for glucose/oxygen EFC operating in (a) human sweat (solid curves) and human sweat with $500 \mu \mathrm{M}$ glucose added (dashed curves), (b) human saliva collected before ingesting a meal (solid curves), after (dashed curves) and after a meal with a further addition of $500 \mu \mathrm{M}$ glucose (dotted curves), showing the current output (left axis) and power output (right axis) as a function of the voltage. The plots were obtained from linear sweep voltammograms recorded at $0.1 \mathrm{mV} \mathrm{s}$.

Table 1 Detailed characteristics of the EFC operating in different biofluids.

\begin{tabular}{lccc} 
Biofluid & OCV $(\mathrm{mV})$ & $\begin{array}{c}\text { Current at } 0.5 \mathrm{~V} \\
\left(\mu \mathrm{A} \mathrm{cm}^{-2}\right)\end{array}$ & $\begin{array}{c}\text { Current at } 0.2 \mathrm{~V} \\
\left(\mu \mathrm{A} \mathrm{cm}^{-2}\right)\end{array}$ \\
\hline Sweat & $580 \pm 5$ & $0.53 \pm 0.05$ & $6.0 \pm 5.1$ \\
Sweat $+500 \mu \mathrm{M}$ glucose & $614 \pm 17$ & $0.94 \pm 0.17$ & $8.45 \pm 4.10$ \\
Saliva before lunch & $560 \pm 13$ & $0.20 \pm 0.05$ & $5.2 \pm 1.5$ \\
Saliva after lunch & $562 \pm 6$ & $0.40 \pm 0.07$ & $10.4 \pm 3.4$ \\
Saliva after & $596 \pm 7$ & $0.91 \pm 0.20$ & $11.6 \pm 2.3$ \\
lunch $+500 \mu \mathrm{M}$ glucose & & &
\end{tabular}

Results averaged over 3-5 different EFCs for each measurement were used to estimate the statistical parameters.

oxidation of different fuels at different potentials; glucose at high potential and glucose in combination with other compounds at low potential. Similar observations were also made in our previous investigations in human tears, which also contain very low amounts of glucose [13]. By adding a small amount of glucose to the sample (dotted curves in Figure 2a) the performance of the EFC was enhanced, with an average OCV of $614( \pm 17) \mathrm{mV}$ and an average current output of $0.94 \mu \mathrm{A}( \pm 0.17) \mathrm{cm}^{-2}$ at $0.5 \mathrm{~V}$ and $8.45( \pm 4.10) \mu \mathrm{A} \mathrm{cm} \mathrm{cm}^{-2}$ at $0.2 \mathrm{~V}$. A large variation between different electrodes was observed at $0.2 \mathrm{~V}$ (Table 1), which can likely be explained by a variation in the coverage of the AuNP layer. Modification with thiols and enzyme serves to protect the underlying surface and reduces the electrocatalytic oxidation occurring due to the supporting layer, whereas exposed AuNPs lead to higher currents at low voltage.

The miniature glucose/oxygen EFC was able to generate an average power output of $0.26( \pm 0.03) \mu \mathrm{W} \mathrm{cm} \mathrm{cm}^{-2}$ in pure sweat and $0.47( \pm 0.08) \mu \mathrm{W} \mathrm{cm}{ }^{-2}$ in sweat spiked with $500 \mu \mathrm{M}$ glucose at $0.5 \mathrm{~V}$. The fact that an addition of $500 \mu \mathrm{M}$ glucose almost generates twice the power output illustrates the very low glucose concentration present in the original sample. At $0.2 \mathrm{~V}$ both samples exhibited similar behavior, with a significantly higher power output ranging between 1.2 and $1.7 \mu \mathrm{W} \mathrm{cm}{ }^{-2}$. When the EFC was operated continuously for $10 \mathrm{~h}$ at $0.2 \mathrm{~V}$ (i.e. roughly at maximum power) in sweat with added glucose, an average of $65( \pm 8) \%$ of the power output remained.
These results can be expected to vary significantly if other donors, collection methods, or areas of collections are used. Differences in sweat composition reflect differences in experimental conditions (exercise, heat exposure, or combination thereof), sweating durations, sweat rate, and sample collection method [19]. The major sweat glands on the human body are the eccrine glands, covering most of the skin and responsible for thermoregulation. Sweat on the skin surface is a mixture of sweat constituents excreted by sweat glands, as well as by-products of the skin surface maturation and skin peeling processes and metabolism by bacteria [11]. The sweat rate is not uniform over the skin surface with large variations for both sweat rate and constituent concentrations between different regions for the same subject [20, 21]. When collecting sweat by scraping from the back, the sweat sample will inevitably contain contamination from epidermal components and certain evaporation will occur, changing the composition of the sweat from the pure sweat excreted from eccrine glands. Among others, this means the sample will contain slightly higher amounts of glucose than pure sweat. Different collection methods can be used in order to reduce the contamination of the sample [11], however, the use of such methods was not relevant in our studies, since that would not correspond to the conditions at which a power generating EFC placed on the skin would operate. Nevertheless, our results clearly show that a glucose/oxygen EFC can be used to generate power from human sweat, and a small change in glucose concentration significantly increases the power at high voltage.

\subsection{Miniature EFC Operating in Human Saliva}

Similar to sweat, saliva contains rather low amounts of glucose with a large variation in reported values, from 30 up to $100 \mu \mathrm{M}$ [22-24]. The composition of saliva can vary significantly depending on oral health and dietary regime [22]. Closely following ingestion of food significantly higher amounts of glucose in the saliva have been observed, up to around $400 \mu \mathrm{M}$ (from originally $\sim 30 \mu \mathrm{M}$ ) [24]. In addition to 
glucose, saliva contains a multitude of other compounds, such as other carbohydrates, urea, different amino acids, peptides, and proteins, as well as lipids. The $\mathrm{pH}$ normally range between 6.2 and 7.6 (average 6.7) [22]. To investigate the change in the performance of the EFC during these widely changing conditions, measurements were performed both in saliva collected before lunch (solid curves Figure 2b) and shortly following lunch (dashed curves Figure 2b). Furthermore, the glucose concentration of the saliva sample collected after ingestion was further increased by an addition of $500 \mu \mathrm{M}$ glucose (dotted curves Figure $2 \mathrm{~b}$ ).

A typical response for the miniature glucose/oxygen EFC operating in human saliva is shown Figure $2 b$ (and displayed as power vs. current in SI, Figure S3B) and corresponding characteristics in Table 1, displaying a similar current response as that observed for measurements in sweat. At high voltage the current output is very low, due to the small amounts of glucose in the samples, whereas it sharply increases at low voltage due to electrooxidation of other compounds than glucose at the AuNP supporting layer. For saliva collected before lunch, the EFC recorded an OCV of around $560( \pm 13) \mathrm{mV}$ with an average current output of 0.20 $( \pm 0.05) \mu \mathrm{A} \mathrm{cm}^{-2}$ at $0.5 \mathrm{~V}$ and $5.2( \pm 1.5) \mu \mathrm{A} \mathrm{cm} \mathrm{cm}^{-2}$ at $0.2 \mathrm{~V}$. Notably, saliva samples collected after lunch displayed a twofold increase in the current output at $0.5 \mathrm{~V}$, with a further two-fold increase at the addition of $500 \mathrm{mM}$ glucose (Table 1). This corresponds to an average power output of 0.1 $( \pm 0.03), 0.2( \pm 0.04)$, and $0.45( \pm 0.10) \mu \mathrm{W} \mathrm{cm} \mathrm{cm}^{-2}$ at $0.5 \mathrm{~V}$ in saliva collected before a meal, after a meal, and with addition of $500 \mu \mathrm{M}$ glucose, respectively, roughly increasing the power output two-fold between each type of saliva sample. This is expected, since the glucose concentration in saliva varies significantly before and after eating, and illustrates that the power output of the EFC in the high voltage region is heavily affected by the glucose concentration. The power output at low voltage $(0.2 \mathrm{~V})$ was quite similar but with a large variation, ranging from $c a .1 .0-2.3 \mu \mathrm{W} \mathrm{cm}{ }^{-2}$. When the EFC was operated continuously for $10 \mathrm{~h}$ at $0.2 \mathrm{~V}$ (i.e. roughly at maximum power) in saliva collected after lunch with added glucose, an average of $60( \pm 15) \%$ of the power output remained.

\subsection{EFCs for Applications in Sweat and Saliva}

The results presented above demonstrate that a glucose/ oxygen EFC can operate in human sweat and saliva, with at least around $50 \%$ of the current output remaining after $10 \mathrm{~h}$ of continuous operation. We have previously investigated the stability of a similar EFC in human blood, plasma, and tears, displaying a similar performance over time, in contrast to simple buffers, where over $80 \%$ of the current output remained [25]. It is difficult to predict the exact cause of the deactivation of the EFC in physiological fluids, which contain many different organic and inorganic compounds that can affect the bioelectrocatalytic activity of both the bioanode and especially the biocathode, viz. chloride ions, uric and ascorbic acids, cells, and a multitude of different proteins. The chosen type of measurement could also in itself affect the apparent stability, as potentiostatic tests have been shown to cause more rapid degradations than galvanostatic tests [26].

The power output at high voltage is due to oxidation of glucose, and is heavily dependent on the concentration in the sample. We also previously examined the EFC in complex buffers and human lachrymal liquid, showing that the power output of the EFC is limited by the comparatively poor performance of the bioanode [13]. The presence of compounds oxidizable by the supporting layer, which are available in tears as well as in sweat and saliva at various concentrations, reduces the onset voltage of the biocathode, and hence reduce the overall voltage of the EFC [13]. The resulting power output is rather limited, due to the performance of the bioanode at the low glucose concentrations present in saliva and sweat. More efficient glucose oxidizing bioanodes have been reported, e.g. based on mediators [6], although the design employed in this study allows for a simple design without potentially toxic mediators and ensures a low onset potential for the oxidation of glucose. The voltage of the EFC is important, since most modern semiconductor based transistors require a voltage of at least $0.5 \mathrm{~V}$ for proper performance [27], although devices exist that can operate at very low voltage [28]. Even though the power output is low, given the fast paced development of modern electronics, such as a wireless autonomous system with an average power consumption of only $5.3 \mathrm{nW}$ being reported [29], an EFC as described herein can still be useful. By integrating an EFC with, e.g. sensing and radio electronics, wireless self-contained (i.e. wireless and self-powered) biosensing devices could be fabricated.

At the high voltage region of the EFC, where the bioanode is the limiting electrode, if the current is limited only by the biofluid production and the EFC is able to utilize all fuel available, the electrical power, which can be attained in sweat and saliva, can be calculated using the very simple equation $P=n F U C v$, where $n$ - number of electrons transferred from one molecule fuel, $F$ - the Faraday constant, $U$ - voltage, $C-$ fuel concentration, $v$ - biofluid production rate. Considering the different parameters in the equation in turn; the biooxidation of glucose is a two-electron transfer process, and maximum power output from utilizing glucose was achieved roughly at $U=0.5 \mathrm{~V}$. Since the fuel concentration may vary significantly, a broad range of concentrations were taken into account, viz. from 5.6 to $2,200 \mu \mathrm{M}$ glucose in sweat and 30 $400 \mu \mathrm{M}$ glucose in saliva. The sweat production rate can vary significantly depending on level of activity, heat exposure, and body area, ranging from almost zero up to $14 \mu \mathrm{L} \mathrm{min} \mathrm{m} \mathrm{cm}^{-2}$ (considering a square centimeter area of the skin) [30]. The un-stimulated salivary flow rate can range from 40 to $400 \mu \mathrm{L} \mathrm{min}{ }^{-1}$ (taking into account all glands in the oral cavity) depending on numerous factors, such as degree of dehydration, body position, smoking, etc. [22]. These broad ranges were taken into account in the calculations for the respective biofluid. The power would naturally also be affected by the actual design of the EFC, if sweat from a larger area could be utilized, the potential power output would increase. Vice 

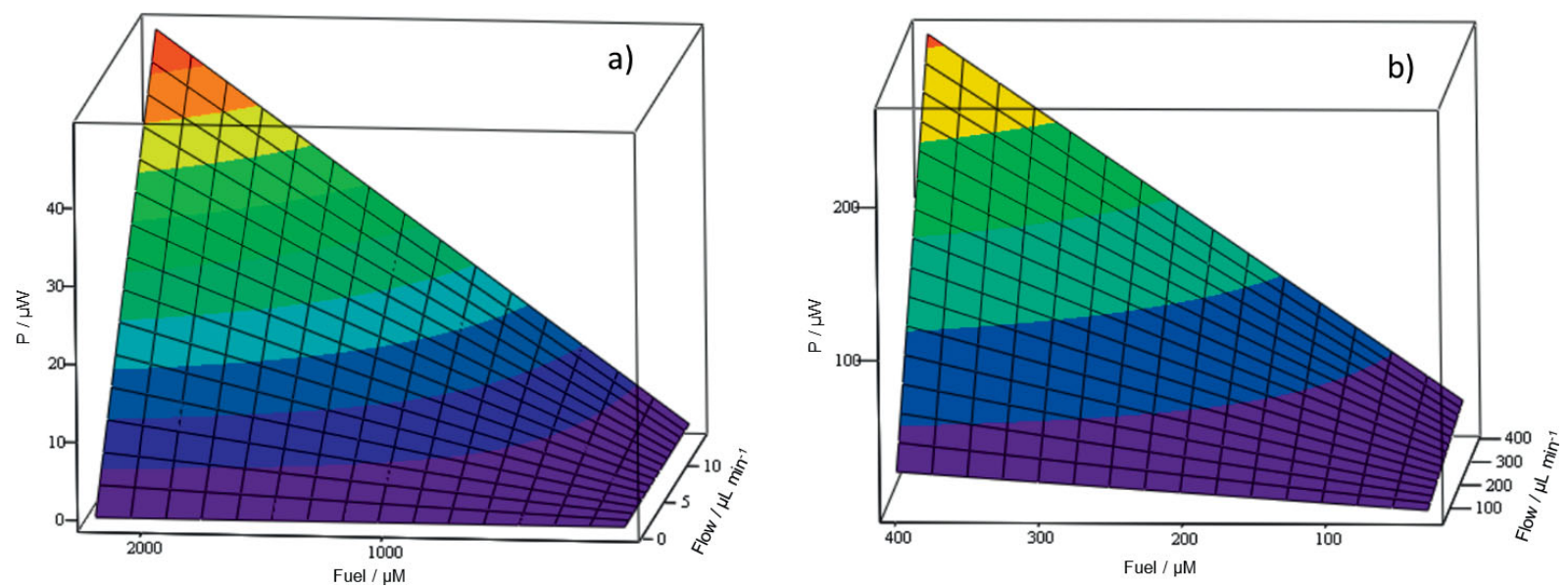

Fig. 3 Result of theoretical calculation of the power output (P) of an EFC in (a) human sweat and (b) human saliva utilizing glucose as fuel, when varying the fuel concentration (Fuel) and biofluid flow rate (Flow).

versa, if only parts of the saliva secreted in the oral cavity could be used to extract power from, the power output would be reduced. The results for both sweat and saliva are shown in Figure $3 a$ and $b$, suggesting power ranges of $0-50 \mu \mathrm{W}$ and $2-260 \mu \mathrm{W}$ in human sweat and saliva, respectively. The range is very wide, depending on the large variation in glucose concentration and the flow rate of the biofluids. Under certain conditions, such as e.g. profuse sweating, the EFC can generate significant power, whereas the power output will be very low under other conditions. This was also very clear, when measuring the power output in saliva before and after lunch (cf. curves in Figure $2 b$ ), where the increased glucose concentration after the meal ingestion significantly affected the power output. The EFC could potentially also be employed as a sensing unit by itself, considering the large change in power output in the high-voltage region depending on the available amount of glucose. Since the devices would be operating in sweat or saliva, designed biodevices would be noninvasive. It should, however, be stressed that the very large variation in composition of samples of saliva and sweat might complicate such measurements.

\section{Conclusion}

We present experimental proof that direct electron transfer based, membrane-and mediator-less EFCs can produce electrical energy from glucose present in human sweat and saliva, where the power was strongly influenced by the amount of glucose in the specific sample. This type of biodevices could potentially be used in non-invasive autonomous biodevices, which could provide a great benefit for biomedical monitoring. Work is currently ongoing in our laboratory to increase the performance of the EFC and as the next step to design flexible and/or transparent EFCs from biocompatible polymeric nanostructured materials.

\section{Acknowledgements}

The authors thank Amano Enzyme Inc. for the kind gift of Amano $3 \mathrm{MvBOx}$ preparation and Dr. Roland Ludwig (BOKU-University of Natural Resources and Life Sciences, Vienna, Austria) for homogeneous $\mathrm{CtCDH}$. The work has been supported financially by the European Commission (FP7 project NMP4-SL-2009-229255 and PEOPLE-2013-ITN607793), Knowledge Foundation (KK stiftelsen, Biofilmsresearch centre for biointerfaces), and Malmö University. D.P., T.A., and L.L. acknowledge the financial support from the Russian Foundation for Basic Research (14-04-32235), the Gustaf Th Ohlsson foundation, and the Swedish Laryng Foundation, respectively.

\section{References}

[1] S. C. Barton, J. Gallaway, P. Atanassov, Chem. Rev. 2004, $104,4867$.

[2] A. Heller, Phys. Chem. Chem. Phys. 2004, 6, 209.

[3] I. Willner, Y. M. Yan, B. Willner, R. Tel-Vered, Fuel Cells 2009, 9, 7 .

[4] B. D. Ratner, A. S. Hoffman, F. J. Schoen, J. E. Lemons, Biomaterials Science, 2nd Ed., Elsevier Academic Press, San Diego, 2004.

[5] V. Andoralov, M. Falk, B. Suyatin Dmitry, M. Granmo, J. Sotres, R. Ludwig, O. Popov Vladimir, J. Schouenborg, Z. Blum, S. Shleev, Sci. Rep. 2013, 3, 3270.

[6] A. Zebda, S. Cosnier, J. P. Alcaraz, M. Holzinger, A. Le Goff, C. Gondran, F. Boucher, F. Giroud, K. Gorgy, H. Lamraoui, P. Cinquin, Sci. Rep. 2013, 3, 1516.

[7] F. C. P. F. Sales, R. M. Iost, M. V. A. Martins, M. C. Almeida, F. N. Crespilho, Lab Chip 2013, 13, 468.

[8] J. A. Castorena-Gonzalez, C. Foote, K. MacVittie, J. Halamek, L. Halamkova, L. A. Martinez-Lemus, E. Katz, Electroanalysis 2013, 25, 1579.

[9] S. Duun, R. G. Haahr, O. Hansen, K. Birkelund, E. V. Thomsen, J. Micromech. Microeng. 2010, 20, 075020/1. 
[10] W. Jia, G. Valdes-Ramirez, A. J. Bandodkar, J. R. Windmiller, J. Wang, Angew. Chem. Int. Ed. 2013, 52, 7233.

[11] J. Moyer, D. Wilson, I. Finkelshtein, B. Wong, R. Potts, Diabetes Technol. Ther. 2012, 14, 398.

[12] C. Li, Y. Chen, W. Chen, P. Huang, H. Chu, in ISWC 2013, Zurich, Switzerland, 2013, pp. 41.

[13] M. Falk, V. Andoralov, Z. Blum, J. Sotres, D. B. Suyatin, T. Ruzgas, T. Arnebrant, S. Shleev, Biosens. Bioelectron. 2012, 37, 38.

[14] C. Dawes, Int. J. Chronobiol. 1974, 2, 253.

[15] X. Wang, M. Falk, R. Ortiz, H. Matsumura, J. Bobacka, R. Ludwig, M. Bergelin, L. Gorton, S. Shleev, Biosens. Bioelectron. 2012, 31, 219.

[16] A. B. Stefaniak, C. J. Harvey, Toxicol. in Vitro 2006, 20, 1265.

[17] C. J. Harvey, R. F. LeBouf, A. B. Stefaniak, Toxicol. in Vitro 2010, 24, 1790.

[18] T. Vidakovic-Koch, I. Ivanov, M. Falk, S. Shleev, T. Ruzgas, K. Sundmacher, Electroanalysis 2011, 23, 927.

[19] T. Verde, R. J. Shephard, P. Corey, R. Moore, J. Appl. Physiol. Respir. Environ. Exercise Physiol. 1982, 53, 1540.

[20] M. J. Patterson, S. D. Galloway, M. A. Nimmo, Exp. Physiol. 2000, 85, 869 .
[21] V. P. Kutyshenko, M. Molchanov, P. Beskaravayny, V. N. Uversky, M. A. Timchenko, PLoS One 2011, 6, e28824.

[22] M. Edgar, C. Dawes, D. O'Mullane, Saliva and oral health, 3rd Ed., British Dental Association, London, 2004.

[23] C. Jurysta, N. Bulur, B. Oguzhan, I. Satman, M. Yilmaz Temel, J. Malaisse Willy, A. Sener, J. Biomed. Biotechnol. 2009, 2009, 430426.

[24] A. W. Dynesen, A. Bardow, A. Astrup, B. Petersson, J. J. Holst, B. Nauntofte, Am. J. Clin. Nutr. 2008, 87, 12.

[25] M. Falk, Z. Blum, S. Shleev, Electrochim. Acta 2012, 82, 191.

[26] A. Shrier, F. Giroud, M. Rasmussen, S. D. Minteer, J. Electrochem. Soc. 2014, 161, H244.

[27] R. Sharpeshkar, Ultra Low Power Bioelectronics: Fundamentals, Biomedical Applications, and Bio-Inspired System, Cambridge University Press, Cambridge, 2010.

[28] J.Sun, B. Zhang, H. E. Katz, Adv. Funct. Mater. 2011, 21, 29.

[29] G. Chen, H. Ghaed, R.-u. Haque, M. Wieckowski, Y. Kim, G. Kim, D. Fick, D. Kim, M. Seok, K. Wise, D. Blaauw, D. Sylvester, A 1 Cubic Millimeter EnergyAutonomous Wireless Intraocular Pressure Monitor in Proc. ISSCC (Ed. S. B. D. 17.6), 2011.

[30] K. Sato, W. H. Kang, K. Saga, K. T. Sato, J. Am. Acad. Dermatol. 1989, 20, 537. 